\title{
ATIVIDADE FÍSICA E MODULAÇÃO DO RISCO CARDIOVASCULAR
}

PHYSICAL ACTIVITY AND MODULATION OF CARDIOVASCULAR RISK

ACTIVIDAD FÍSICA Y MODULACIÓN DEL RIESGO CARDIOVASCULAR

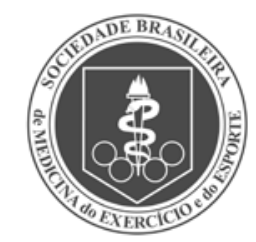

Artigo Original

Original Article

Artículo Original
Marcelo Cichocki' (Médico)

Karen Parron Fernandes ${ }^{1}$ (Dentista)

Daniellen Cristhine Castro-Alves' ${ }^{1}$ (Biomédica)

Marcus Vinicius de Matos Gomes' (Biomédico)

1. Universidade Norte do Paraná (UNOPAR). Londrina, PR, Brasil.

\section{Correspondência:}

Marcus Vinicius de Matos Gomes. Centro de Ciências Biológicas e da Saúde, Universidade Norte do Paraná (UNOPAR). Av. Paris 675, Jardim Piza, Londrina, PR, Brasil. 86041-120. mvmgomes@gmail.com

\section{RESUMO}

Introdução: As doenças cardiovasculares são responsáveis por alto grau de morbidade e mortalidade na população mundial. Em populações saudáveis, a estimativa do risco cardiovascular tem sido calculada a partir de modelos de predição derivados de estudos prospectivos e observacionais. O escore de Framingham é um algoritmo tradicionalmente utilizado como estratégia primária de prevenção de doenças cardiovasculares em pessoas assintomáticas em um período de 10 anos. Para a determinação desse escore são considerados como fatores de risco os níveis séricos de colesterol total, colesterol HDL, pressão sistólica, diabetes mellitus, tabagismo e idade dos pacientes. Objetivo: Avaliar se a prática de atividade física é uma variável importante capaz de modular o risco obtido pelo escore de Framingham. Métodos: Foram considerados dados de 1004 indivíduos, divididos em adultos (18 a 59 anos) e idosos (> 60 anos), usando o software Statistical Package for Social Sciences (SPSS) para as análises estatísticas e adotando-se o nível de significância de $5 \%(P<0,05)$ para todos os testes aplicados. Resultados: A correlação entre os fatores idade, sexo, tabagismo, hipertensão arterial e níveis séricos de HDL e o escore de Framingham foi confirmada tanto no grupo adulto como no idoso. Adicionalmente, nossas análises demonstraram correlação estatisticamente significativa entre a prática de atividade física e o risco calculado por esse escore, ficando evidente que indivíduos adultos ou idosos que praticam atividade física moderada ou intensa apresentam menor risco de desenvolver doença cardiovascular num período de 10 anos. Conclusão: Nossos dados demonstram que a prática de atividade física moderada ou intensa pode modular o escore de Framingham e assim como idade, sexo, tabagismo, hipertensão arterial e níveis séricos de colesterol total e HDL, deve ser considerada entre os fatores de risco de doenças cardiovasculares.

Descritores: doenças cardiovasculares; fatores de risco; atividade física.

\section{ABSTRACT}

Introduction: Cardiovascular diseases are responsible for a high degree of morbidity and mortality in the world population. In healthy populations, the estimated cardiovascular risk has been calculated from predictive models derived from prospective and observational studies. The Framingham score is an algorithm traditionally used as a primary strategy to prevent cardiovascular disease in asymptomatic patients over a period of 10 years. Some risk factors are considered to determine this score such as serum levels of total cholesterol, HDL cholesterol, systolic blood pressure, diabetes mellitus, smoking and age of patients. Objective: To evaluate if the practice of physical activity is an important variable capable of modulating cardiovascular risk obtained by the Framing ham score. Methods: Data from 1004 individuals, divided into adults (18 to 59 years) and elderly (>60 years) were considered using Statistical Package for Social Sciences (SPSS) software for statistical analyses and the significance level was established at 5\% $(P<0.05)$ for all tests applied. Results: The correlation between age, sex, smoking, hypertension and serum levels of HDL and the Framingham score was confirmed in both the adult and elderly group. Additionally, our analyses demonstrate a statistically significant correlation between the practice of physical activity and the risk calculated by this score, making it clear that adult or elderly individuals who practice moderate or intense physical activity have a lower risk of developing cardiovascular disease within 10 years. Conclusion: Our data demonstrate that moderate or intense physical activity can modulate the Framingham score and, as well as the age, sex, smoking, hypertension and serum levels of total cholesterol and HDL cholesterol, should be considered as a risk factor for cardiovascular diseases.

Keywords: cardiovascular diseases; risk factors; physical activity.

\section{RESUMEN}

Introducción: Las enfermedades cardiovasculares son responsables de un alto grado de morbilidad y mortalidad en la población mundial. En las poblaciones sanas, la estimación del riesgo cardiovascular se ha calculado a partir de modelos de predicción derivados de estudios prospectivos y de observación. La puntuación de Framingham es un algoritmo usado tradicionalmente como una estrategia primaria para la prevención de la enfermedad cardiovascular en personas asintomáticas en un perío do de 10 años. Para determinar esta puntuación son considerados factores de riesgo como los niveles de colesterol total, colesterol HDL, presión arterial sistólica, diabetes mellitus, tabaquismo yedad de los pacientes. Objetivo: Evaluar si la actividad física es una variable importante que puede modular el riesgo obtenido por la puntuación de Framingham. Métodos: Se consideraron los datos de 1004 sujetos, divididos en adultos (18-59 años) y ancianos (> 60 años) utilizando el software Statistical Package for Social Sciences (SPSS) para el análisis estadístico, siendo 
adoptado el nivel de significación estadística del $5 \%(P<0,05)$ para todas las pruebas. Resultados: La correlación entre los factores edad, sexo, tabaquismo, hipertensión y niveles séricos de HDL y la puntuación de Framingham fue confirmada tanto en el grupo de adultos como en los ancianos. Además, nuestro análisis mostró una correlación estadísticamente significativa en tre la actividad física y el riesgo calculado por esta puntuación, dejando claro que los adultos o ancianos que practican actividad física moderada o intensa tienen menor riesgo de desarrollar enfermedad cardiovascular en un periodo de 10 años. Conclusión: Nuestros datos demuestran que la práctica de actividad física moderada o intensa puede modular la puntuación de Framingham y, asícomo edad, sexo, tabaquismo, hipertensión y los niveles séricos de colesterol total y HDL, debe ser considerada entre los factores de riesgo de enfermedad cardiovascular.

Descriptores: enfermedades cardiovasculares; factores de riesgo; actividad física.

\section{INTRODUÇÃO}

As doenças cardiovasculares são a principal causa de morte na população adulta em todo o mundo. De acordo com a American Heart Association', os fatores de risco comumente associados à doença cardiovascular são: dislipidemia, obesidade, hipertensão arterial, diabetes e tabagismo. Além destes, o risco de eventos cardiovasculares aumenta com a idade, assim como, a prevalência da síndrome metabólica².

Mudanças no estilo de vida são entusiasticamente recomendadas na prevenção de doenças cardiovasculares e, a despeito da mencionada associação com o número de óbitos na população mundial, dados consistentes da literatura demonstram que a não exposição aos fatores de risco como o tabagismo, dieta inadequada, sedentarismo, bem como a obesidade e a hipertensão arterial podem fazer com que o número de mortes ligadas às doenças cardiovasculares diminua significativamente ${ }^{3-7}$.

Na prática clínica, a avaliação do risco cardiovascular pode ser estimada a partir da utilização de escores, como o escore de Framingham, o qual se utiliza de dados relativos à exposição dos pacientes aos principais fatores de riscos cardiovasculares, para determinar o risco absoluto do desenvolvimento de doença coronariana no período de dez anos ${ }^{8,9}$.

O escore de Framingham é atualmente o estimador de risco cardiovascular mais utilizado em todo o mundo ${ }^{10}$. No entanto não há evidência na literatura de que o sedentarismo (ou a prática de exercício físico) também possa ser um fator importante em sua modulação.

Os efeitos positivos obtidos pela prática de atividade física sob a saúde, de uma forma geral, são inquestionáveis. Entre as implicações ao sistema cardiovascular pode-se incluir a redução do colesterol total (CT), lipoproteína de baixa densidade (LDL-C), triglicerídeos (TG), pressão arterial, melhora nos níveis séricos de colesterol e lipoproteína de alta densidade (HDL-C), além da contribuição para o controle glicêmico, para as respostas fisiológicas relacionadas ao aumento da demanda metabólica, e ao aporte de oxigênio associado aos gastos musculares ${ }^{11}$. Segundo a American College of Sports Medicine e American Heart Association preconiza-se que indivíduos sadios devam realizar atividade física moderada por no mínimo 30 minutos por cinco dias na semana, ou 20 minutos de atividade intensa por três dias na semana a fim de promover e manter a saúde ${ }^{7,1}$.

No entanto, apesar do consenso quanto aos efeitos benéficos diretos e indiretos proporcionados pela prática de atividade física, no tocante aos parâmetros cardiovasculares, é escassa a literatura que possibilite a extrapolação da influência desta prática sob a modulação do risco calculado na avaliação clínica de pacientes.

Neste contexto, avaliamos no presente estudo, se a prática de atividade física é uma variável importante capaz de modular o risco verificável pelo escore de Framingham de um indivíduo desenvolver uma doença cardiovascular.

\section{MATERIAL E MÉTODOS}

O presente estudo foi aprovado pelo Comitê de Ética em Pesquisa da Universidade Norte do Paraná (UNOPAR) (CAAE 12736413.7.0000.0108) e consistiu em uma análise observacional, descritiva e com delineamento transversal baseado em dados de prontuário eletrônico dos pacientes.

Foram incluídos dados coletados em consultas médicas realizadas em pacientes adultos (>18 anos) no período que compreende o mês de fevereiro de 2006 e setembro de 2012.

No total participaram 1004 indivíduos, os quais foram divididos em 683 adultos (com idade entre 18 e 59 anos) e 321 idosos (idade superior a 60 anos).

Entre os dados dos pacientes utilizados nas análises, estão incluídos:

a) Dados demográficos: sexo; idade (em anos completos, categorizados em faixas etárias: 18 a 30, >30 a 59 anos).

b) Hábitos de vida: tabagista (sim ou não).

c) Pressão arterial: A pressão arterial foi aferida por método indireto, utilizando-se um esfignomanômetro (Durashock DS44 Tycos - Welch Allyn, Skaneateles Falls, New York, USA) aneroide e adequação do manguito à circunferência braquial, após 10 minutos de repouso na posição sentada. Foram registradas as pressões sistólicas (PAS) e diastólica (PAD), correspondentes às fases I e V dos sons de Korotkoff, respectivamente.

Foram definidos como hipertensos, segundo os critérios estabelecidos pelas VI Diretrizes Brasileiras de Hipertensão Arterial em 2010, o indivíduo que apresentou pressão sistólica $\geq 140 \mathrm{mmHg}$ e/ou pressão diastólica $\geq 90 \mathrm{mmHg}$ ou indivíduos que eram já hipertensos em uso regular de medicação anti-hipertensiva.

d) Dislipidemia: foram mensurados pela avaliação do LDL (lipoproteínas de baixa densidade) segundo a Equação de Friedwald, HDL (lipoproteínas de alta densidade) e TG (Triglicerídeos), sendo obtidos os dados de prontuário medidos em $\mathrm{mg} / \mathrm{dl}$; conforme previamente preconizado no Consenso Brasileiro de Dislipidemia, 2010'2.

e) Atividade Física: mensurada segundo as recomendações da ACSM (American College Sports of Medicine) e da American Heart Association para atividade física e saúde pública em adultos?

Foram definidos como: atividade física leve: $<3,0$ MetS (por ex. caminhar, lavar pratos e pescar); atividade física moderada: 3,0-6,0 MetS (por ex. esporte recreacional, caminhar rápido, lavar janela); atividade física vigorosa: > 6,0 MetS (por ex. correr, trotar e esportes vigorosos).

f) Escore de Risco de Framingham: foram utilizados para o cálculo do risco de evento cardiovascular por este escore as informações referentes à pressão arterial sistólica, colesterol total, idade, o tabagismo e o HDL-colesterol. A análise foi feita observando-se os fatores de risco dentro de uma tabela definida para homens e mulheres, sendo o resultado uma estimativa do risco absoluto de evento cardiovascular em dez anos.

\section{Análise estatística}

Foi utilizado o programa Statistical Package for Social Sciences (SPSS, Chicago, IL, USA) versão 20.0 para análise dos resultados, sendo adotado 
um intervalo de confiança de 95\% e nível de significância de 5\% (P< $0,05)$ para todos os testes aplicados.

As variáveis qualitativas foram apresentadas por meio de tabelas de frequência (absoluta e relativa) enquanto as variáveis quantitativas foram apresentadas por meio de média e desvio-padrão ou mediana e intervalo interquartílico (em caso de distribuição não normal dos dados).

Inicialmente foi analisada a possível associação entre gênero, idade, tabagismo, classificação da hipertensão, perfil lipídico (colesterol total, triglicerídeos, HDL e LDL) e prática de atividade física com o risco cardiovascular (segundo o escore de Framinghan) pela Correlação de Spearman. Posteriormente, foi utilizado um modelo de regressão linear múltipla para avaliar a importância destes fatores independentes na determinação do risco cardiovascular.

\section{RESULTADOS}

Primeiramente os pacientes foram divididos em dois grupos, sendo adultos (18 a 59 anos) e idosos (>60 anos), posteriormente subcategorizados quanto ao gênero (masculino e feminino), tabagismo (sim ou não), hipertensão (sim ou não) e praticantes de atividade física regular (leve, moderada a intensa) (Tabela 1).

De uma forma geral, entre os 683 pacientes adultos 12,4\% apresentaram idade $>30$ anos tendo os demais $87,7 \%$ entre 30 e 59 anos. Aproximadamente $56 \%$ dos indivíduos eram do sexo masculino, 22\% eram tabagistas e 34,7\% hipertensos. Destes 683 pacientes 20,5\% praticavam atividade física leve e 79,5\% atividade moderada a intensa.

Entre os 321 pacientes do grupo de idosos 37,7\% apresentavam idades entre 60 e 70 anos tendo, portanto, os demais, 62,3\% apresentavam idades $>70$ anos. Assim como observado no grupo dos adultos, predominaram no grupo de idosos os indivíduos do sexo masculino (62,3\%), 24,3\% eram tabagistas e 50,8\% hipertensos. Dos 321 pacientes 47,7\% praticavam atividade física leve e 52,3\% atividade física de moderada a intensa.

No tocante ao perfil lipídico os pacientes dos grupos adultos e idosos foram categorizados de acordo com os respectivos níveis séricos de LDL, $\mathrm{HDL}$, triglicérides e colesterol total (Tabela 2).

Tanto para os grupos de pacientes adultos e idosos, aproximadamente 50\% apresentam valores de LDL considerados ótimos ou limítrofes, considerando a mediana de $120 \mathrm{mg} / \mathrm{dl}$ e $113 \mathrm{mg} / \mathrm{dl}$ destas populações, respectivamente. Para as dosagens de HDL, todos os pacientes apresentaram níveis adequados. A maioria (aproximadamente 75\%) dos indivíduos apresentaram dosagens consideradas ótimas ou limítrofes para os valores de triglicerídeos, enquanto que no tocante à dosagem de colesterol a maioria dos indivíduos apresentaram valores ótimos.

Os resultados dos 1004 pacientes incluídos neste estudo foram comparados com os preconizados pela Sociedade Brasileira de Cardiologia, em sua V Diretriz Brasileira de Dislipidemia e Prevenção da Aterosclerose, $2013^{13}$

Foi observada correlação estatística inversa entre a prática de atividade física e o escore de Framingham (teste do Qui-quadrado, p<0,0001, Tabela 3) apresentando menor escore os indivíduos que praticavam exercícios regularmente de forma moderada ou intensa tanto em adultos quanto idosos.

Diferença estatisticamente significativa foi observada na população de indivíduos adultos quando correlacionado o escore de Framingham e os fatores de risco idade (correlação direta), gênero (correlação inversa = risco maior para o gênero masculino), tabagismo (correlação direta), hipertensão (correlação inversa), atividade física (correlação inversa), a dosagem de HDL (correlação inversa) e colesterol total (correlação direta) (Tabela 4).

Similarmente, na população de idosos foi observada correlação estatisticamente significativa entre o escore de Framingham e os fatores de risco idade (correlação direta), gênero (correlação inversa = risco maior para o gênero masculino), tabagismo (correlação direta), hipertensão (correlação inversa), atividade física (correlação inversa) e a dosagem de HDL (correlação inversa) e colesterol total (correlação direta) (Tabela 4).

Na análise multivariada (regressão linear múltipla), as variáveis que permaneceram associadas como determinantes do escore de Framingham foram: idade, gênero, tabagismo, hipertensão, e prática de atividade física regular (Tabela 5).

Na análise multivariada (regressão linear múltipla), as variáveis que permaneceram associadas como determinantes do escore de Framingham nesta população de idosos foram: idade, gênero, tabagismo, hipertensão, prática de atividade física regula e níveis plasmáticos de HDL (Tabela 6).

Tabela 1. Caracterização das variáveis independentes no grupo de indivíduos adultos e idosos.

\begin{tabular}{|c|c|c|c|c|c|}
\hline \multicolumn{3}{|c|}{ Adultos } & \multicolumn{3}{|c|}{ Idosos } \\
\hline Variáveis & $\begin{array}{c}\text { Frequência } \\
\text { absoluta } \\
\text { (n) }\end{array}$ & $\begin{array}{l}\text { Frequência } \\
\text { relativa (\%) }\end{array}$ & Variáveis & $\begin{array}{c}\text { Frequência } \\
\text { absoluta } \\
\text { (n) }\end{array}$ & $\begin{array}{l}\text { Frequência } \\
\text { relativa (\%) }\end{array}$ \\
\hline \multicolumn{3}{|c|}{ Idade } & \multicolumn{3}{|c|}{ Idade } \\
\hline 18 a 30 anos & 85 & 12,4 & 60-70 anos & 121 & 37,7 \\
\hline$>30$ a 59 anos & 598 & 87,6 & $>70$ anos & 200 & 62,3 \\
\hline Total & 683 & 100 & Total & 321 & 100 \\
\hline \multicolumn{3}{|c|}{ Gênero } & \multicolumn{3}{|c|}{ Gênero } \\
\hline Masculino & 382 & 55,9 & Feminino & 121 & 37,7 \\
\hline Feminino & 301 & 44,1 & Masculino & 200 & 62,3 \\
\hline Total & 683 & 100 & Total & 321 & 100 \\
\hline \multicolumn{3}{|c|}{ Tabagismo } & \multicolumn{3}{|c|}{ Tabagismo } \\
\hline Sim & 150 & 22 & Sim & 78 & 24,3 \\
\hline Não & 533 & 78 & Não & 243 & 75,7 \\
\hline Total & 683 & 100 & Total & 321 & 100 \\
\hline \multicolumn{3}{|c|}{ Hipertensão } & \multicolumn{3}{|c|}{ Hipertensão } \\
\hline Sim & 237 & 34,7 & Sim & 163 & 50,8 \\
\hline Não & 446 & 65,3 & Não & 158 & 49,2 \\
\hline Total & 683 & 100 & Total & 321 & 100 \\
\hline \multicolumn{3}{|c|}{ Atividade física regular } & \multicolumn{3}{|c|}{ Atividade física regular } \\
\hline Leve & 140 & 20,5 & Leve & 153 & 47,7 \\
\hline $\begin{array}{c}\text { Moderada/ } \\
\text { Intensa }\end{array}$ & 543 & 79,5 & $\begin{array}{c}\text { Moderada/ } \\
\text { Intensa }\end{array}$ & 168 & 52,3 \\
\hline Total & 683 & 100 & Total & 321 & 100 \\
\hline
\end{tabular}

Tabela 2. Caracterização das variáveis do perfil lipídico no grupo de indivíduos adultos e idosos.

\begin{tabular}{c|c|c|c|c|c|c|c}
\hline \multicolumn{4}{c|}{ Adultos } & \multicolumn{4}{c}{ Idosos } \\
\hline Variáveis & Mediana & $\begin{array}{c}\mathbf{1 0}^{\mathbf{2}} \\
\text { Quartil }\end{array}$ & $\begin{array}{c}\mathbf{3}^{\mathbf{0}} \\
\text { Quartil }\end{array}$ & Variáveis & Mediana & $\begin{array}{c}\mathbf{1 0}^{\mathbf{2}} \\
\text { Quartil }\end{array}$ & $\begin{array}{c}\mathbf{3}^{\mathbf{2}} \\
\text { Quartil }\end{array}$ \\
\hline $\mathrm{LDL}$ & 120 & 93 & 144 & $\mathrm{LDL}$ & 113 & 87 & 141 \\
\hline $\mathrm{HDL}$ & 47 & 41 & 55 & $\mathrm{HDL}$ & 50 & 41 & 59 \\
\hline $\mathrm{TG}$ & 129 & 86 & 188 & $\mathrm{TG}$ & 122 & 90 & 179 \\
\hline $\mathrm{CT}$ & 196 & 169 & 228 & $\mathrm{CT}$ & 195 & 164 & 224 \\
\hline
\end{tabular}

Tabela 3. Caracterização das correlações entre as variáveis do estudo e o risco cardiovascular avaliado pelo Escore de Framingham.

\begin{tabular}{c|c|c|c|c|c|c|c}
\hline \multicolumn{3}{|c|}{ Adultos } & \multicolumn{4}{c}{ Idosos } \\
\hline $\begin{array}{c}\text { Prática de } \\
\text { atividade } \\
\text { física } \\
\text { regular }\end{array}$ & \multicolumn{2}{|c|}{ Risco cardiovascular } & $\begin{array}{c}\text { Prática de } \\
\text { atividade } \\
\text { física } \\
\text { regular }\end{array}$ & \multicolumn{2}{c}{ Risco cardiovascular } \\
\hline & Baixo & Moderado & Alto & & Baixo & Moderado & Alto \\
\hline Leve & 99 & 28 & 13 & Leve & 43 & 61 & 49 \\
\hline & $70,70 \%$ & $20,00 \%$ & $9,30 \%$ & & $28,10 \%$ & $39,90 \%$ & $32,00 \%$ \\
\hline Moderada & 259 & 48 & 12 & Moderada & 55 & 55 & 14 \\
\hline & $81,20 \%$ & $15,00 \%$ & $3,80 \%$ & & $44,40 \%$ & $44,40 \%$ & $11,30 \%$ \\
\hline Intensa & 149 & 9 & 0 & Intensa & 10 & 3 & 0 \\
\hline & $94,30 \%$ & $5,70 \%$ & $0,00 \%$ & & $76,90 \%$ & $23,10 \%$ & $0,00 \%$ \\
\hline
\end{tabular}




\section{DISCUSSÃO}

Corroborando a literatura, no presente estudo a correlação entre o aumento da idade, gênero, presença de tabagismo, gravidade da hipertensão, aumento dos níveis plasmáticos de colesterol total e LDL com o aumento do escore de Framingham foi confirmada em adultos e idosos. Além disso, demonstramos uma correlação estatisticamente significativa entre os valores de risco obtidos pelo escore de Framingham e a prática de atividade física realizada.

Um número crescente de estudos tem considerado a prática da atividade física como um importante fator na diminuição de riscos e mortes

Tabela 4. Caracterização das correlações entre as variáveis do estudo e o escore de Framingham.

\begin{tabular}{|c|c|c|c|c|c|}
\hline \multicolumn{3}{|c|}{ Adultos } & \multicolumn{3}{|c|}{ Idosos } \\
\hline Variáveis & $\begin{array}{l}\text { Coeficiente } \\
\text { de } \\
\text { correlação } \\
\text { (rS) }\end{array}$ & $p$ & Variáveis & $\begin{array}{l}\text { Coeficiente } \\
\text { de } \\
\text { correlação } \\
\text { (rS) }\end{array}$ & $p$ \\
\hline Idade & 0,37 & 0,0001 & Idade & 0,34 & 0,0001 \\
\hline Gênero & $-0,23$ & 0,0001 & Gênero & $-0,421$ & 0,001 \\
\hline Tabagismo & 0,32 & 0,0001 & Tabagismo & 0,259 & 0,001 \\
\hline Hipertensão & $-0,27$ & 0,0001 & Hipertensão & $-0,27$ & 0,0001 \\
\hline $\begin{array}{c}\text { Atividade física } \\
\text { moderada/ } \\
\text { Intensa }\end{array}$ & $-0,22$ & 0,0001 & $\begin{array}{c}\text { Atividade física } \\
\text { moderada/ } \\
\text { Intensa }\end{array}$ & $-0,19$ & 0,002 \\
\hline $\mathrm{HDL}$ & $-0,14$ & 0,001 & $\mathrm{HDL}$ & $-0,33$ & 0,0001 \\
\hline CT & 0,15 & 0,0001 & CT & 0,05 & 0,37 \\
\hline
\end{tabular}

Tabela 5. Análise multivariada (regressão linear múltipla) entre as variáveis e o escore de Framingham na população adulta.

\begin{tabular}{c|c|c|c}
\hline \multirow{2}{*}{ Variáveis } & $\begin{array}{c}\text { Coeficientes } \\
\text { não ajustados }\end{array}$ & $\begin{array}{c}\text { Coeficientes } \\
\text { ajustados }\end{array}$ & $\boldsymbol{p}$ \\
\cline { 2 - 4 } & Beta & Beta & \\
\hline Idade & 0,013 & 0,265 & 0,0001 \\
\hline Gênero & $-0,24$ & $-0,247$ & 0,0001 \\
\hline Tabagismo & $-0,355$ & $-0,293$ & 0,0001 \\
\hline Hipertensão & 0,06 & 0,146 & 0,0001 \\
\hline HDL & $-0,003$ & $-0,084$ & 0,017 \\
\hline CT & 0,002 & 0,158 & 0,001 \\
\hline $\begin{array}{c}\text { Atividade física } \\
\text { moderada/Intensa }\end{array}$ & $-0,059$ & $-0,082$ & 0,02 \\
\hline
\end{tabular}

Tabela 6. Análise multivariada (Regressão linear múltipla) entre as variáveis e o escore de Framingham na população idosa.

\begin{tabular}{c|c|c|c}
\hline \multirow{2}{*}{ Variáveis } & $\begin{array}{c}\text { Coeficientes } \\
\text { não ajustados }\end{array}$ & $\begin{array}{c}\text { Coeficientes } \\
\text { ajustados }\end{array}$ & \multirow{2}{*}{$\boldsymbol{p}$} \\
\cline { 2 - 3 } & Beta & Beta & \\
\hline Idade & 0,04 & 0,36 & 0,0001 \\
\hline Gênero & 0,53 & 0,34 & 0,0001 \\
\hline Tabagismo & $-0,45$ & $-0,26$ & 0,0001 \\
\hline Hipertensão & 0,1 & 0,2 & 0,0001 \\
\hline CT & 0,002 & 0,129 & 0,006 \\
\hline HDL & $-0,017$ & $-0,279$ & 0,0001 \\
\hline Atividade física & $-0,235$ & $-0,18$ & 0,0001 \\
moderada/Intensa & & &
\end{tabular}

por doenças cardiovasculares ${ }^{14-18}$. A atividade física consiste em parte integrante no tratamento do paciente acometido por doenças coronárias, também desempenha um importante papel em sua prevenção ${ }^{19}$.

Sua ação mais direta está relacionada com a melhora do perfil lipídico, estímulo para produção de substâncias vasodilatadoras e melhora da função endotelial. Na prevenção primária atua combatendo os principais fatores de risco desencadeadores das doenças cardiovasculares, além disso, a prática regular de atividade física e o estilo de vida ativa são métodos eficazes para controlar os níveis de ansiedade ${ }^{20}$.

De fato, os benefícios do exercício físico já são bem conhecidos na literatura quanto à prevenção e controle dos fatores de riscos. Necessitam, todavia, de uma visão mais abrangente e um aprofundamento específico de sua relação com a fisiopatologia das doenças. Assim, é imprescindível identificar os fatores e conhecer dos efeitos do exercício físico em modular os riscos cardiovasculares, com a finalidade preponderante de na conduta médica e no tratamento de indivíduos cardiopatas. Na prática clínica, o escore de Framingham é atualmente o estimador de risco cardiovascular mais utilizado para o desenvolvimento de doença coronariana no período de dez anos ${ }^{10}$.

No presente estudo os indivíduos foram categorizados, de acordo com a prática de atividade física, em: a) praticantes de atividade física leve: indivíduos que relataram capacidade de realizar atividade de pouca intensidade como caminhar, lavar pratos e pescar, b) moderada: indivíduos que relataram capacidade de praticar esporte recreacional, caminhar rápido, lavar janela e c) intensa: indivíduos que relataram capacidade de praticar esportes vigorosos, correr, trotar.

Entre os pacientes adultos 20,5\% praticavam atividade física leve e $79,5 \%$ atividade moderada a intensa, enquanto que no grupo de idosos $47,7 \%$ praticavam atividade física leve e 52,3\% atividade física de moderada a intensa.

Com base nos dados obtidos no presente estudo, demonstramos que a prática de atividade física é um potencial modulador do risco de Fram/ingham e que, assim como os fatores idade, gênero, tabagismo, presença de hipertensão arterial e níveis séricos de colesterol total e $\mathrm{HDL}$, deve ser considerado na prática clínica para a obtenção do risco de um indivíduo desenvolver doenças cardiovasculares.

Além disso, nossos dados demonstram que o benefício modulador sobre o escore de Framingham somente é obtido pelos indivíduos que praticam atividade física moderada a intensa e de forma regular, e que apenas a prática de atividades leves não apresenta benefício sobre os riscos de um indivíduo desenvolver doenças cardiovasculares.

Em suma, sugerimos que a variável prática de atividade física é um potencial modulador do risco de Framingham e que, deve ser considerada juntamente com as variáveis idade, gênero, tabagismo, presença de hipertensão arterial e níveis séricos de colesterol total e HDL, na prática clínica do cálculo do risco do indivíduo desenvolver uma doença cardiovascular nos próximos 10 anos.

\section{CONCLUSÃO}

Nossos resultados permitem concluir que a prática de atividade física por adultos e idosos, somente tem um efeito sobre o risco cardiovascular se realizado regularmente de forma moderada ou intensa.

Todos os autores declararam não haver qualquer potencial conflito de interesses referente a este artigo.

CONTRIBUIÇÕES DOS AUTORES: Cada autor contribuiu individual e significativamente para o desenvolvimento deste estudo. MC (000-0003-1923-1928)* e MVMG (00000002-5408-8883)* delinearam o estudo. MC avaliou os pacientes, analisou os prontuários e coletou os dados clínicos. KPF (0000-0002-1276-4900) avaliou os dados das análises estatísticas. MC, KPF, DCCA (0000-0001-9620-5227) e MVMG realizaram a pesquisa bibliográfica, contribuíram na redação e revisão do manuscrito. *ORCID (Open Researcher and Contributor ID). 


\section{REFERÊNCIAS}

1. American College of Sports Medicine Position Stand and American Heart Association. Recommendations for cardiovascular screening, staffing, and emergency policies at health/fitness facilities. Med Sci Sport Exer. 1998;30(6):1009-18.

2. Zimmet P, Magliano D, Matsuzawa Y, Alberti G, Shaw J. The metabolic syndrome: a global public health problem and a new definition. J Atheroscler Thromb. 2005;12(6):295-300.

3. Ockene JS, Miller NH. Cigarette smoking, cardiovascular disease, and stroke: a statement for healthcare professionals from the American Heart Association. American Heart Association Task Force on Risk Reduction. Circulation. 1997; 96(9):3243-7.

4. Kannel WB. Risk stratification in hypertension: new insights from the Framingham. Am J Hypertens. 2000;13(1 Pt 2): 35-10.

5. Stamler J, Vaccaro O, Neaton JD, Wentworth D. Diabetes, other risk factors, and 12-yr cardiovascular mortality for men screened in the multiple risk factor intervention trial. Diabetes Care. 1993;16(2):434-44

6. Warren TY, Barry V, Hooper SP, Sui X, Church TS, Blair SN. Sedentary behaviors increase risk of cardiovascular disease mortality in men. Med Sci Sports Exerc. 2010;42(5):879-85.

7. Ford ES, Kohl HW, Mokdad AH, Ajani UA. Sedentary behavior, physical activity, and the metabolic syndrome among US adults. Obes Res. 2005; 13: 608-14.

8. Anderson KM, Wilson PWF, Odell PM, KannelWB. An updated coronary risk profile. A statement for health professionals. Circulation. 1991;83(1):356-62.

9. Kannel WB, Feinleib M, McNamara PM, Garrison RJ, Castelli WP. An investigation of coronary heart disease in families. The Framingham offspring study. Am J Epidemiol. 1979;110(3):281-90.

10. Hu G, Lakka TA, Kilpeläinen TO, Tuomilehto J. Epidemiological studies of exercise in diabetes prevention. Appl Physiol Nutr Metab. 2007;32((3):583-95.
11. Abete I, Goyenechea E, Zulet MA, Martinez JA. Obesity and metabolic syndrome: potential benefit from specific nutritional components. Nutr Metab Cardiovasc Dis. 2011;21(Suppl 2):B1-15.

12. Sociedade Brasileira de Cardiologia. Sociedade Brasileira de Hipertensão; Sociedade Brasileira de Nefrologia. VI Diretrizes Brasileiras de Hipertensão. Arq Bras Cardiol. 2010;95(Suppl 1):1-51.

13. Sociedade Brasileira de Cardiologia. V Diretriz Brasileira de Dislipidemia e Prevenção de Aterosclerose. Arq. Bras de Cardiol. 2013;101(4 Suppl.1):1-20

14. Haskell WL, Lee IM, Pate RR, Powell KE, Blair SN, Franklin BA. Physical activity and public health: updated recommendation for adults from the American College of Sports Medicine and the American Heart Association. Circulation. 2007;116(9):1081-93.

15. Zhang L, Qin LQ, Liu AP, Wang PY. Prevalence of risk factors for cardiovascular disease and their associations with diet and physical activity in suburban Beijing, China. J Epidemiol. 2010;20(3):237-43.

16. Reddigan Jl, Ardern Cl, Riddell MC, Kuk JL. Relation of physical activity to cardiovascular disease mortality and the influence of cardiometabolic risk factors. Am J Cardiol. 2011;108(10):1426-31.

17. Luke A, Dugas LR, Durazo-Arvizu RA, Cao G, Cooper RS. Assessing physical activity and its relationship to cardiovascular risk factors: NHANES 2003-2006. BMC Public Health. 2011;11:387.

18. Ryoo JH, Cho SH, Kim SW. Prediction of risk factors for coronary heart disease using Framingham risk score in Korean men. PLos ONE. 2012;7(9):e45030

19. Vuori IM, Lavie CJ, Blair SN. Physical activity promotion in the health care system. Mayo Clin Proc. 2013;88(12):1446-61.

20. Yorston LC, Kolt GS, Rosenkranz R. Physical Activity and physical function in older adults: the 45 and up study. J Am Geriatr Soc. 2012;60(4):719-25. 